\title{
Spin accumulation in ballistic Rashba bar
}

\author{
J. Yao and Z. Yang** \\ Surface Physics Laboratory (National Key Laboratory), Fudan University, Shanghai, 200433, China
}

\begin{abstract}
We propose an analytic model to study intrinsic spin polarization effect in a ballistic Rashba bar with two semi-infinite leads. The wave functions expanded with plane waves in Rashba bar are required to satisfy boundary conditions at both longitudinal and transverse interfaces. We find outof-plane spin Hall accumulation effect can be induced in the Rashba bar even with large dimensions by injecting unpolarized current from the lead. The longitudinal in-plane spin Hall effect, however, becomes obscure in large-size sample. An interesting direction-flipping of the out-of-plane spin accumulation is predicted by altering the Rashba coupling strength.
\end{abstract}

PACS Number: 72.25.Dc,73.23.Ad, 85.75.Nn

Introduction: An external electric field can be expected to induce a transverse spin current in non-magnetic semiconductors or result in out-of-plane spin accumulation near the edges of semiconductor films due to the spinorbit coupling (SOC) of electron. It is the so-called spin Hall effect (SHE) 1]. Very recently, two independent groups have reported the experimental observation of SHE 2, 3], giving evidence of the existence of significant SOC in semiconductors. Such new effect of SOC creates a new way to manipulate electron spins by means of an electric field, not magnetic field, that may have great potential applications in future spintronics. SHE was first proposed by D'yakonov and V. I. Perel' in 1971[4], then Hirsch [1] and Zhang [5]. The existence of SHE effect in p-type semiconductors and n-type semiconductors in two-dimensional heterostructures was predicted by Murakami et al. [6] and Sinova et al. [7], respectively. However, the microscopic origin of SHE still has controversial explanations. The discussion of extrinsic or intrinsic origin currently attracts many scientists. [8, 9, 10, 11, 12, 13, 14, 15, 16, 17] This letter focuses on the study of spin accumulation in Rashba SOC system in ballistic region, since ballistic samples usually with small dimensions are always desirable for future electronic device applications. References 18, 19, 20, 21] investigated spin accumulation in ballistic system with Rashba interaction. Based on discrete tight-binding model and Landauer-Büttiker formula, Nikolić et al. 18] numerically demonstrated opposite spin accumulation near the two edges of the two dimensional semiconductor structure. The phenomena is qualitatively similar to the experimental observations 2, 3]. Very recently, Wang et al. 19] reported current induced local spin polarization due to Rashba SOC in a two dimensional narrow strip, where no lead is considered to attach to the Rashba strip. They concluded that out-of-plane spin polarization is important mainly in systems with mesoscopic sizes, and it appears not to be associated with the SHE in bulk samples.

In this paper, we propose an analytic model to study intrinsic spin polarization effect in a Rashba bar con- nected to two semi-infinite leads. The geometry studied is similar to that proposed by Nikolić et al. 18]. We, however, don't use the discrete tight-binding model, but a continuous model to find out its exact solution to Schrödinger equation. The method we developed can properly deal with the coupling between channels in the Rashba bar. Therefore, the behavior of spin accumulation as a function of the width of the Rashba bar can be studied systematically. We find spin accumulation can be induced in Rashba bar by injecting unpolarized current from the lead. With the increase of the width of the Rashba bar, the peaks of spin accumulation shift towards the two lateral edges and the oscillations within the bar decrease, indicating the polarization becomes more prominent in the ballistic bar. This conclusion is very different from that obtained by Wang et al. 19]. Meanwhile, we also report an interesting direction-flipping of the out-of-plane spin accumulation by altering the spinorbital coupling strength, which may be considered as a signature of SHE in ballistic region.

Theoretical Approach: We consider a 2DEG Rashba bar with two opposite interfaces connected to two semiinfinite ideal leads respectively along $x$ direction. To simplify the calculation (not losing the essential physics), the leads and the Rashba bar are assumed to have the same widths in $y$ direction. And open boundary condition is applied at the two lateral edges of the strip. An electron wave is injected from the right lead to the left one and crossing the middle Rashba bar. We solve separately Schrödinger equations of the three regions. The Hamiltonian of the structure is described by

$$
\mathbf{H}=\left[-\frac{\hbar^{2}}{2 m^{*}} \nabla^{2}+V(y)\right]\left(\begin{array}{ll}
1 & 0 \\
0 & 1
\end{array}\right)+\frac{\alpha}{\hbar}(\vec{\sigma} \times \vec{p})_{z},
$$

where $m^{*}$ is effective mass of electrons and $\alpha$ the Rashba SOC strength that can be tuned by gate voltage 22. $\alpha=0$ in leads, and $\alpha \neq 0$ in the Rashba bar. $V(y)$ is the confined potential in $y$ direction. For the open boundary, it is simply taken as $V(y)=0$, $(0<y<b) ; V(y)=\infty,(y \leq 0 ; y \geq b)$, where $b$ is the width of the strip. The total wave func- 
tion contains two components, $\psi(x, y)=\psi_{1}(x, y)\left(\begin{array}{l}1 \\ 0\end{array}\right)+$ $\psi_{2}(x, y)\left(\begin{array}{l}0 \\ 1\end{array}\right)$, where $\psi_{1}, \psi_{2}$ can generally expressed as $\psi_{1}(x, y)=\sum_{k_{n}, k_{x}} C_{n}\left(k_{x}\right) \chi_{n}(y) e^{i k_{x} x}, \psi_{2}(x, y)=$ $\sum_{k_{n}, k_{x}} D_{n}\left(k_{x}\right) \chi_{n}(y) e^{i k_{x} x}$, where $\chi_{n}(y)=\sin \left(k_{n} y\right), n=$ $1,2,3 \ldots$. In the formula, $k_{n}=n \pi / b$ and $n$ is the channel number. They are the wave functions that satisfy the hard-wall boundary conditions in $y$ direction, $\left.\psi(x, y)\right|_{y=0 ; y=b}=0$. With above wave functions, the Schrödinger equation is simplified as:

$$
\begin{aligned}
& E_{n}\left(k_{x}\right) C_{n}\left(k_{x}\right)+i \alpha \sum_{n^{\prime}}\left[\delta_{n n^{\prime}} k_{x}-\Delta_{n, n^{\prime}} k_{n^{\prime}}\right] D_{n^{\prime}}\left(k_{x}\right)=0, \\
& E_{n}\left(k_{x}\right) D_{n}\left(k_{x}\right)-i \alpha \sum_{n^{\prime}}\left[\delta_{n n^{\prime}} k_{x}+\Delta_{n, n^{\prime}} k_{n^{\prime}}\right] C_{n^{\prime}}\left(k_{x}\right)=0
\end{aligned}
$$

where $\quad E_{n}\left(k_{x}\right)=e_{n}\left(k_{x}\right)-E, \quad e_{n}\left(k_{x}\right)=\hbar^{2}\left(k_{x}^{2}+k_{n}^{2}\right) / 2 m^{*}$, $n, n^{\prime}=1,2,3 \ldots, N$, and $\Delta_{n, n^{\prime}}=\frac{2}{b} \int_{0}^{b} \sin \left(k_{n} y\right) \cos \left(k_{n^{\prime}} y\right) d y$, $\left(n \neq n^{\prime}\right) . \mathrm{N}$ is the total number of channels existing in the Rashba bar and dependent on the width $b$ of the strip and the energy $E$ of incident electrons. At fixed energy $E$, the total number $N$ is large with large width $b$. The terms containing $\Delta_{n, n^{\prime}}$ in Eq.(2) and (3) indicate explicitly the mixing interactions between different subbands due to the Rashba coupling and the boundaries existing in $y$ direction. Our investigation reveals that no spin accumulation can be observed for the case of single channel in the bar, while the accumulation appears in multi-channel cases. This implies the mixing terms between subbands play an essential role in forming the spin accumulation effect.

From Eq.(2) and (3), we have a polynomial equation of $k_{x}$ with the highest power of $4 N$. Therefore, $4 N$ solutions of $k_{x}^{(j)}(j=1 \rightarrow 4 N)$ can be obtained from the equation for any fixed $E$ and $N$. In the case of single channel, where the SOC lifts the degenerate spin up and down states at $\pm k_{x}$ into 4 different wave vectors $\pm k_{x 1}$ and $\pm k_{x 2}$. In general, $N$ subbands would produce $4 N$ different $k_{x}^{(j)}$. Each $k_{x}^{(j)}$ corresponds to an eigenfunction $\widehat{\Phi}_{N}^{(j)}$ which has a length of $2 N$. All the coefficients $\left\{C_{n}\left(k_{x}^{(j)}\right), D_{n}\left(k_{x}^{(j)}\right)\right\}$ in $\widehat{\Phi}_{N}^{(j)}$ are determined up to a normalized constant. And the wave function in Rashba region with eigenvalue $E$ must be a linear combination of $4 N$ eigenfunctions $\widehat{\Phi}_{N}^{(j)}, j=1,2, \cdots, 4 N$. The combined coefficients can be denoted by coefficients $\Lambda\left(k_{x}^{(j)}\right)$. Therefore, in Rashba region, there are totally $4 N$ coefficients $\left\{\Lambda\left(k_{x}^{(j)}\right)\right\}$ to be determined. If the Rashba bar is at an equilibrium state, $4 N$ eigenfunctions in Rashba bar have equivalent weight. There will be no spin polarization in the sample since Rashba interaction does not change the time-reversal symmetry. The time-reverse symmetry, however, is broken in the bar if there is steady current flowing along $x$ direction. And spin polarization will occur in the sample. Suppose an unpolarized incident electron wave injected from the right lead. There are $2 \mathrm{~N}$ coefficients describing reflected waves in the right lead with respect to $N$ channels for spin up and down states. Similarly, additional $2 N$ coefficients are introduced to express the transmitted waves in the left lead. Therefore, there are totally $8 N$ coefficients to be determined. They can be solved by boundary conditions at the two interfaces.

The interfaces between leads and Rashba bar denoted as $x=0, a$ are simplified by a $\delta$ potential 23. $V_{0} \delta\left(x-x_{0}\right), x_{0}=0, a$ ( $a$ is the length of the Rashba bar). The continuous conditions of wave functions and particle current crossing each interface, say at $x=0$, yield equations: $\left.\psi_{L}(x)\right|_{x=0^{-}}=\left.\psi_{\text {mid }}(x)\right|_{x=0^{+}}$ and $\left.\frac{\widehat{P}_{L x}}{2 m_{L}^{*}} \psi_{L}(x)\right|_{x=0^{-}}-\left.\frac{\widehat{P}_{m i d x}}{2 m_{m i d}^{*}} \psi_{m i d}(x)\right|_{x=0^{+}}=i \frac{V_{0}}{\hbar} \psi_{L}(0)$, where $\widehat{P}_{L x}=(\hbar / i) \partial / \partial x$, and $\widehat{P}_{\text {mid } x}=(\hbar / i) \partial / \partial x-$ $\left(\alpha m^{*} / \hbar\right) \sigma_{y}$ 23, 24]. It has been assumed that there's no spin-flip across the interfaces 23]. By means of the orthonormality of $\chi_{n}$, each subband of spin-up or -down state would satisfy above boundary conditions. Therefore, for the structure with $N$ channels, two interfaces along $x$ direction will yield $8 N$ equations, the same as the number of coefficients to be determined. All the coefficients in the wave functions thus can be exactly solved. With the wave function obtained, the local spin polarization $\left\langle S_{i}(x, y)\right\rangle=\frac{\hbar}{2}\left\langle\psi(x, y)\left|\sigma_{i}\right| \psi(x, y)\right\rangle$ is straightforward to be calculated in the Rashba region.

Results and Discussion: In the calculation, we focus on the linear region and simply assume that the Fermi levels and the effective masses of electrons in Rashba bar and two leads are the same. They are taken as $10 \mathrm{meV}$ and $0.04 \mathrm{~m}_{e}$, typical values in 2DEG 22, 25]. The current injection has been normalized to one non-polarized electron wave for each channel. Meanwhile, we do the statistical average for the spin polarization of each incident electron wave with energy $E$ in each channel. The spin polarizations of $\left\langle S_{z}\right\rangle,\left\langle S_{x}\right\rangle$ and $\left\langle S_{y}\right\rangle$ with different numbers of channel are demonstrated in Fig.1, where average integrals along $x$ direction are taken. Note that the scale in the horizontal axis is normalized in order to compare explicitly the trends. The channel numbers of 3,6 , and 9 correspond to the widths of about 120, 210, and $300 \mathrm{~nm}$, respectively. The length of Rashba bar in the direction of current flow is fixed at $210 \mathrm{~nm}$. It can be found that these sizes are within the phase-coherent length $\left(L_{\phi} \lesssim 0.4 \mu-1 \mu\right) 22$, 26. Experimentally permitted value of $2.9 \times 10^{-11} \mathrm{eVm}[22,27]$ is taken for the strength of Rashba coupling. In the case of 3 channels, $\left\langle S_{z}\right\rangle$ has two high peaks with inverse symmetry distribution along y direction and there are small oscillations in the middle of the sample. With the increase of the channel number, the two high peaks become narrow and shift gradually to the lateral edges with the inside oscillations tending to be smoothed out. The phenomenon of out-of-plane spin polarization with opposite sign near the two edges is very much similar to the prediction of spin Hall effect by Hirsch [1] and recent experimental observations [2, 3]. We also calculated spin polariza- 


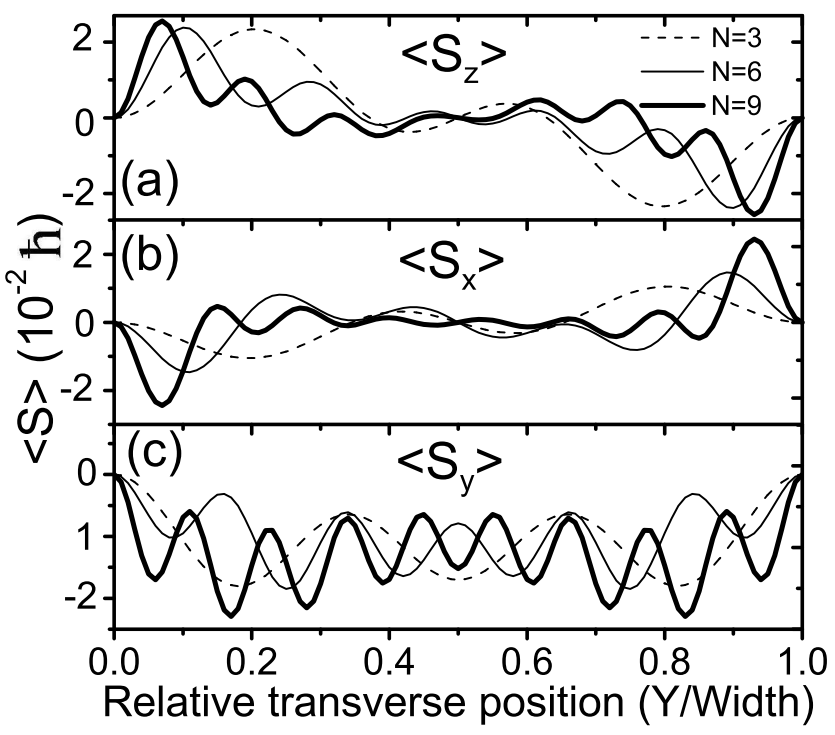

FIG. 1: The average spin accumulation of $\left\langle S_{z}\right\rangle$ (a), $\left\langle S_{x}\right\rangle$ (b) and $\left\langle S_{y}\right\rangle$ (c) as a function of the lateral position in the Rashba bar. The spin polarizations varied with the number of channel are indicated with different curve styles.

tion of the Rashba bar having 20 channels (about 630 $\mathrm{nm}$ in $y$ direction). The envelop of its accumulation has the same behavior and keeps very strong spin Hall accumulation effect. Therefore, the out-of-plane spin accumulation becomes more prominent with the increase of the channel number in the sample, namely, the width of the sample. This tendency is different from the conclusion obtained by Wang et al. 19]. They reported that the out-of-plane polarization increases at small width of sample, decreases, however, at large width with $L \sim 16 / k_{F}$ (about 6 channels). From our result, no evidence is found to support that their such tendency 19 can also exist in the Rashba bar with two leads at least to the width of the bar with 20 channels.

The in-plane spin accumulations of $\left\langle S_{x}\right\rangle$ and $\left\langle S_{y}\right\rangle$ are given in Fig.1(b) and (c), respectively. The distribution of $\left\langle S_{x}\right\rangle$ as a function of transverse position shares the same feature of $\left\langle S_{z}\right\rangle$, also forming SHE effect [1, 2, 3]. With the increase of the channel number, the effect of $\left\langle S_{x}\right\rangle$ also becomes more obvious. For $\left\langle S_{z}\right\rangle$ and $\left\langle S_{x}\right\rangle$, the net total spin polarization is zero because of the inverse symmetry of the distribution along $y$ direction. The spin accumulation of $\left\langle S_{y}\right\rangle$, however, has a uniform sign along the $y$ direction, which gives a net total spin polarization. The net transverse in-plane magnetization induced from unpolarized current can be ascribed to the combined factors of Rashba SOC and time-reversal symmetry breaking in the structures studied.

From Eq. (2) and (3), the behavior of spin polarizations of $\left\langle S_{z}\right\rangle,\left\langle S_{x}\right\rangle$, and $\left\langle S_{y}\right\rangle$ with opposite Rashba strength $(-\alpha)$ 18 can be obtained analytically. In Eq.(2) and (3), an opposite Rashba coefficient $-\alpha$ produces an

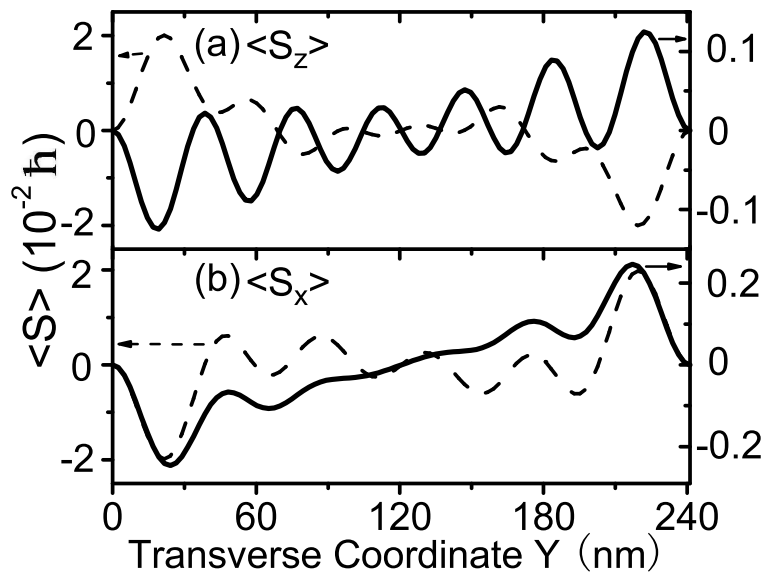

FIG. 2: Spin accumulation of $\left\langle S_{z}\right\rangle$ (a) and $\left\langle S_{x}\right\rangle$ (b) with two different Rashba strengths $\left(2.9 \times 10^{-12} \mathrm{eVm}\right.$, dotted line; $2.9 \times 10^{-11} \mathrm{eVm}$ solid line). The interesting direction-flipping is observed for $\left\langle S_{z}\right\rangle$, while no flipping is obtained for $\left\langle S_{x}\right\rangle$.

opposite set of $D_{n}\left(k_{x}^{j}\right)$, while $C_{n}\left(k_{x}^{j}\right)$ is not varied. Therefore, $\left.\psi_{1}\right|_{-\alpha}=\left.\psi_{1}\right|_{\alpha}$ and $\left.\psi_{2}\right|_{-\alpha}=-\left.\psi_{2}\right|_{\alpha}$ (see Eq.(2) and (3)), which directly yield $\left\langle S_{z}(x, y)\right\rangle_{-\alpha}=\left\langle S_{z}(x, y)\right\rangle_{\alpha}$ and $\left\langle S_{x, y}(x, y)\right\rangle_{-\alpha}=-\left\langle S_{x, y}(x, y)\right\rangle_{\alpha}$. When the direction of Rashba interaction is reversed, the in-plane spin accumulation flips the polarization directions, while the out-ofplane one does not. We demonstrate, however, that in a ballistic SOC strip, the sign of $\left\langle S_{z}\right\rangle$ can be flipped by modulating the SOC strength, as depicted in Fig.2(a). The thick curve in Fig.2(a) shows the spin polarization of $\left\langle S_{z}\right\rangle$ with small Rashba strength. With the increase of the strength to a certain value, opposite peaks near the lateral edges emerge, and the amplitudes magnify gradually while the inside oscillations decrease. The flippingvalue of $\alpha$ is revealed to be inversely proportional to the width of the sample. No flipping, however, is observed for $\left\langle S_{x}\right\rangle$ by modulating the value of Rashba strength in the whole experimental permitted range 22, 27] (see Fig. 2(b)). Since the Rashba interaction strength in a normal 2DEG can be tuned through gate voltage, we suggest a direct experimental detection of the spin-flipping of $\left\langle S_{z}\right\rangle$ crossing a critical value of Rashba SOC.

The spin polarizations for $\left\langle S_{z}(x, y)\right\rangle$ and $\left\langle S_{x}(x, y)\right\rangle$ with long length (about $660 \mathrm{~nm}$ ) of the Rashba bar are demonstrated in Fig. 3 (a) and (b), respectively. With 3 channels in the bar, very obvious period-like variation of the pattern along $x$ is observed for both $\left\langle S_{z}\right\rangle$ and $\left\langle S_{x}\right\rangle$, giving rise to the spin precession in the sample due to the SOC. The rough period of the precession is found to be closely related to the characteristic Rashba length $L_{S O}=\frac{\hbar}{2 m^{*} \alpha}$. The rough period will be shorter if there are more channels in the sample or larger SOC. In Fig. 3(a), the sign of $\left\langle S_{z}\right\rangle$ keeps in period-like variation along $x$ direction, while $\left\langle S_{x}\right\rangle$ flips its sign during the variation (see Fig. 3(b)). This featured pattern of $\left\langle S_{x}\right\rangle$, however, is obscured with the increase of the width of the 
(a) $<\mathrm{S}_{\mathrm{z}}(\mathrm{x}, \mathrm{y})>$

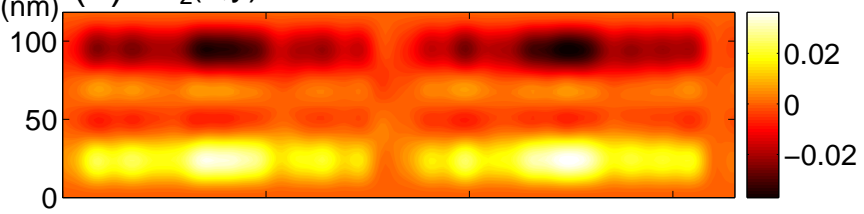

(b) $<S_{x}(x, y)>$

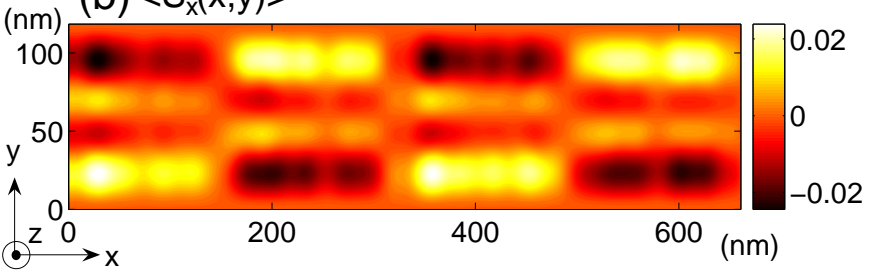

FIG. 3: Contour plots of spin accumulation of out-of-plane $\left\langle S_{z}(x, y)\right\rangle$ (a) and longitudinal in-plane $\left\langle S_{x}(x, y)\right\rangle$ (b) with long length of rashba bar. There are 3 channels in the sample. The Rashba strength used is $2.9 \times 10^{-11} \mathrm{eVm}$.

sample due to having more oscillations along $x$ direction. Therefore, for large dimensions of ballistic bar, the outof-plane SHE can survive, while the longitudinal in-plane spin Hall polarization may not be detectable in the case.

The variation of spin-polarization pattern under opposite bias voltage 2] is predicted. If the center of the Rashba bar is taken as the origin, the wave function should have following symmetry: $\left\langle\psi(x, y)\left|S_{i}\right| \psi(x, y)\right\rangle_{-V}=$ $\left\langle\psi(x, y)\left|P^{\dagger} U^{\dagger} S_{i} U P\right| \psi(x, y)\right\rangle_{V}$, where $P$ is the rotation of $\pi$ angle around $z$ axis in orbital space, and $U$ is $\mathrm{SU}(2)$ rotation with same angle $\pi$ in spin space. It is straightforward to know that $U=\left(\begin{array}{cc}i & 0 \\ 0 & -i\end{array}\right)$. Therefore, following relations can be obtained for the case of reverse bias.

$$
\begin{aligned}
\left\langle\psi(x, y)\left|S_{z}\right| \psi(x, y)\right\rangle_{-V} & =\left\langle\psi(-x,-y)\left|S_{z}\right| \psi(-x,-y)\right\rangle_{V} \\
\left\langle\psi(x, y)\left|S_{x}\right| \psi(x, y)\right\rangle_{-V} & =-\left\langle\psi(-x,-y)\left|S_{x}\right| \psi(-x,-y)\right\rangle_{V} \\
\left\langle\psi(x, y)\left|S_{y}\right| \psi(x, y)\right\rangle_{-V} & =-\left\langle\psi(-x,-y)\left|S_{y}\right| \psi(-x,-y)\right\rangle_{V}
\end{aligned}
$$

Our numerical calculations have exactly confirmed these symmetric relations, which are not completely same as the ones reported by Nikolić et al. 18]. For $\left\langle S_{z}(x, y)\right\rangle$ under reverse bias voltage, the pattern of spin polarization does not just flip its sign [2], but has inverse symmetry compared to the original one (see Fig. 3(a)). It is also an intrinsic feature of SHE in ballistic region.

Summary: We studied intrinsic spin polarization effect in a ballistic Rashba bar by proposing an analytic model. Both the out-of-plane and longitudinal in-plane spin Hall accumulation effects are observed. In the sample with large dimensions, only the out-of-plane spin Hall accumulation can be observed obviously. The transverse in-plane spin accumulation shows same sign across the sample, giving net spin magnetization. This spin nonconservation can be ascribed to the Rashba interaction together with the time-reversal symmetry breaking in the structure. An interesting direction-flipping of the out-of- plane spin accumulation is found by altering the Rashba coupling strength. This phenomenon is suggested to be confirmed by experiment. Some symmetric relations of spin polarizations are predicted.

Acknowledgments: The authors are grateful to ShouCheng Zhang, Fuchun Zhang and Ruibao Tao for very helpful discussion. The work is supported by National Natural Science Foundation with grant No. 10304002 and the Fudan High-end Computing Center.

E-mail address: zyang@fudan.edu.cn.

1 J. E. Hirsch, Phys. Rev. Lett. 83, 1834 (1999).

2 Y. K. Kato, R. C. Myers, A. C. Gossard, and D. D. Awschalom, Science 306, 1910 (2004).

3 J. Wunderlich, B. Kästner, J. Sinova, T. Jungwirth, Phys. Rev. Lett. 94, 047204 (2005).

4 M. I. D'yakonov and V. I. Perel', JETP Lett. 13, 467 (1971).

5 S. Zhang, Phys. Rev. Lett. 85, 393 (2000).

6 S. Murakami, N. Nagaosa, S.-C. Zhang, Science 301, 1348 (2003).

7 J. Sinova, D. Culcer, Q. Niu, N. A. Sinitsyn, T. Jungwirth, and A. H. MacDonald, Phys. Rev. Lett. 92, 126603 (2004).

${ }^{8}$ L. Sheng, D. N. Sheng, and C. S. Ting, Phys. Rev. Lett. 94, 016602 (2005).

9 E. M. Hankiewicz, L. W. Molenkamp, T. Jungwirth, and J. Sinova, Phys. Rev. B 70, 241301 (2004).

10 E. I. Rashba, Phys. Rev. B 68, 241315(R) (2003).

11 J. Schliemann, and D. Loss, Phys. Rev. B 69, 165315 (2004).

12 S-Q. Shen, Phys. Rev. B 70, 081311(R) (2004).

13 J. Inoue, G. E. W. Bauer, and L. W. Molenkamp, Phys. Rev. B 70, 041303(R) (2004).

14 S. Murakami, N. Nagaosa and S.-C. Zhang, Phys. Rev. Lett. 93, 156804 (2004).

15 E. G. Mishchenko, A. V. Shytov, B. I. Halperin, Phys. Rev. Lett. 93, 226602 (2004).

16 D. Culcer, J. Sinova, N. A. Sinitsyn, T. Jungwirth, A. H. MacDonald and Q. Niu, Phys. Rev. Lett. 93, 046602 (2004).

17 S. Zhang and Z. Yang, Phys. Rev. Lett. 94, 066602 (2005).

18 B. K. Nikolić, S. Souma, L. P. Zârbo, and J. Sinova, cond-mat/0412595

19 Q. Wang, L. Sheng, and C. S. Ting, cond-mat/0505576

20 M. Governale and U. Zülicke, Phys. Rev. B 66, 073311 (2002).

21 G. Usaj and C. A. Balseiro, cond-mat/0405065

22 J. Nitta, T. Akazaki, H. Takayanagi, T. Enoki, Phys. Rev. Lett. 78, 1335 (1997).

23 T. Matsuyama, C.-M. Hu, D. Grundler, G. Meier, and U. Merkt, Phys. Rev. B 65, 155322 (2002).

24 U. Zülicke and C. Schroll, Phys. Rev. Lett. 88, 029701 (2002).

25 F. Mireles and G. Kirczenow, Phys. Rev. B 64, 024426 (2001).

26 K. Nomura, J. Sinova, T. Jungwirth, Q. Niu, and A. H. MacDonald, Phys. Rev. B 71, 041304 (2005); S. Souma and B. K. Nikolić, Phys. Rev. Lett. 94, 106602 (2005).

27 G. Engels, J. Lange, Th. Schäpers, H. Lüth, Phys. Rev. B 
55 1958(R) (1997). 